\title{
“El Confesional en Carmeleño. Una ventana a la relación entre franciscanos e indios Rumsen en las misiones de California”.
}

\author{
“The Confesional en Carmeleño. Vintage point to the relationship \\ between Franciscans and Rumsen Indians in the California missions".
}

josé refugio de la torre curiel. Doctor en Historia por la Universidad de California en Berkeley. Sus líneas de investigación comprenden la historia del poblamiento del norte novohispano, historia de la cartografía sobre el norte novohispano, y la historia de los franciscanos en México. Entre sus publicaciones recientes destacan Twilight of the Mission Frontier: Shifting Interethnic Alliances and Social Organization in Sonora, 1768-1855, Stanford y Berkeley, Stanford University Press - The Academy of American Franciscan History, 2012; y el volumen colectivo Expansión territorial y formación de espacios de poder en la Nueva España, Zapopan, El Colegio de Jalisco, 2016.

RESUMEN. En 1770 fray Junípero Serra fundó la misión de San Carlos Borromeo, en las inmediaciones del presidio de Monterrey (hoy Monterey), en California. Al año siguiente, la misión fue trasladada a su ubicación definitiva en el río Carmelo, entre los indios Rumsen. Mediante la transcripción y análisis de un "Confesional en Carmeleño" preparado por un anónimo misionero del Colegio de San Fernando de México (posiblemente asociado a la época de fray Junípero Serra o Juan Crespi), el artículo da a conocer un valioso testimonio de una de las lenguas nativas de California según quedó registrada por los franciscanos a finales del siglo XVIII. Este manual es un texto bilingüe, castellano-rumsen, el cual además de su valor lingüístico y etnográfico ofrece algunas pistas sobre las formas en que los misioneros identificaban algunas prácticas culturales de la población local.

PALABRAS CLAVE. Manuales para confesores; Monterey (California); confesionarios; Junípero Serra; Juan Crespi; Indios de California

ABSTRACT. In 1770, fray Junípero Serra founded mission San Carlos Borromeo, on the surroundings of the Monterey presidio in California. The following year, the mission was relocated to its permanent site on the banks of the Carmel River, among the Rumsen Indians. Through the transcription and analysis of a "Confesional en Carmeleño" (or Manual for confession in Carmeleño) compiled by an anonymous missionary from the College of San Fernando de Mexico (probably related to the times of Junípero Serra or Juan Crespi), the article makes available imporant fragments of one of California's native langues, as recorded by one of the late-eighteenth century Franciscan missionaries. This manual is a bilingual Castilian-Rumsen text; in addition to its linguistic and ethnographic value, the document shades light on the ways in which the missionaries identified some native cultural practices.

KEYWORDS. Manuals for confessors, Monterey (California), confesionarios, Junipero Serra, Juan Crespi; California Indians

INFORMACIÓN DEL ARTÍCULO

Recibido: 10 de julio de 2017

Aprobado: 21 de junio de 2018

Correo para correspondencia: cucodelatorre@gmail.com

Agradecimientos: El presente trabajo forma parte del proyecto de investigación "El gran norte novohispano-mexicano en el tiempo y el espacio: estudios sobre poblaciones y territorios en perspectiva comparada" (CONACyT, Investigación Científica Básica 2015-1, proyecto 250624). 
INTRODUCCIÓN

En la tradición católica, a partir del cuarto Concilio de Letrán (1215-1216) distintos teólogos y tratadistas discurrieron sobre la forma en que sacerdotes y penitentes se debían disponer para participar en el sacramento de la confesión, ${ }^{1}$ con lo que comenzaron a aparecer distintos manuales para confesores "para proporcionar al confesor y al penitente las preguntas y las respuestas relacionadas con el sacramento". ${ }^{2}$ Se trataba de manuscritos que bajo diversas denominaciones (Summae confessorum, Summa de casibus poenitenciae, Confessional, o Modus confitendi) abordaban estas materias en latín, y gradualmente en distintas lenguas vernáculas. Vale la pena mencionar que por circunscribirse a la forma de preparar la confesión del penitente, este tipo de guías se diferenciaba de los catecismos, las guías de doctrina o los manuales para sacerdotes, los cuales abordaban materias más generales.

La circulación del libro impreso sería un paso importante en la difusión de los manuales para confesores. Al menos así lo sugiere el caso español, donde uno de los primeros libros impresos en el siglo XV sería el Modus confitendi o manual para la confesión, compuesto por el obispo lusitano Andrés de Escobar (1348-1439); en este caso, se trataba de un texto impreso hacia 14721473 el cual gozaría de "gran difusión en su época". ${ }^{3}$ Durante el siglo XVI, en el contexto de la Contrarreforma, los manuales para confesión "experimentaron una amplia popularidad” en Europa occidental, en una época en la cual la "penitencia cambió de un rito comunitario diseñado para mantener la paz de

1 La sesión 21 del concilio imponía, para aquellos fieles que hubieran alcanzado "los años de discresión", la obligación de confesarse por lo menos una vez al año, de manera individual y con su propio sacerdote. Orrego (2014, p. 60). Este autor, a su vez, se basa en la transcripción de los documentos del concilio publicada por Denzinger y Hünermann (1999, pp. 361-362).

2 Christensen (2013, p. 91).

3 De los Reyes Gómez (2004, p. 27). El Modus confitendi habría sido escrito por Escobar hacia 1415, y publicado de manera póstuma. Otras obras del mismo teólogo portugués sobre la confesión serían una Confessio maior (ca. 1415-1416), y el Lumen Confessorum (1429). 
la comunidad, a un proceso en el cual el individuo se reconciliaba con Dios en las profundidades de su alma". ${ }^{4}$ En este contexto particular, obras como el Manual de confesores y penitentes, de Martín de Azpilcueta, o la Suma de doctrina cristiana, de Constantino Ponce de la Fuente, o las Instrucciones a los Confesores, de San Carlos Borromeo, se convertirían en ejemplos de la literatura de este tipo que ejercería notable influencia en el desarrollo de esta misma tradición en la Nueva España tras la llegada de los primeros religiosos de las ordenes mendicantes. ${ }^{5}$

Cuando los franciscanos llegaron a la Nueva España, pusieron énfasis en la importancia de la confesión auricular, y desde luego, introdujeron el sacramento de la penitencia. ${ }^{6}$ Desde fechas tempranas, los miembros de las órdenes mendicantes se dieron a la tarea de adaptar a las necesidades de la nueva Iglesia la estructura de las guías de confesión que circulaban en Europa. ${ }^{7}$ Para la segunda mitad del siglo xvi, la obra evangelizadora en la Nueva España contaba ya con una bien asentada tradición de preparación de textos bilingües para la explicación de la doctrina cristiana y para guiar a sacerdotes y penitentes en el confesionario; no está de más recordar que tanto el Concilio de Trento como el Concilio III Provincial Mexicano impulsarían estas prácticas al reforzar la doctrina del sacramento de la penitencia. ${ }^{8}$

4 McLaughlin (2008, p. 63).

5 Para un estudio sobre la abundante muestra de manuales de confesión que circularon en Europa durante los primeros años de la imprenta, véase Aspell (2008, pp. 431-447).

6 "Cada domingo por la tarde juntaban a los indios que habían de confesarse en la semana, les hacían un examen acerca de la doctrina [..., luego una plática acerca de la necesidad y eficacia de la penitencia y las tres partes del sacramento, por lo que toca al penitente: contrición, confesión y satisfacción. Seguía a esto una serie de advertencias prácticas sobre el modo de acercarse a la confesión. La mañana del día en que iban a confesarse se les decía la lista de las faltas que puede cometer un hombre. Durante la Cuaresma, periodo reservado especialmente a la confesión, los religiosos intérpretes se consagraban de modo exclusivo a la confesión de los indios [....]”. Ricard (2005, p. 209).

7 Murillo Gallegos (2009, pp. 28-35); Moreno (1984, p. 17).

8 El Concilio de Trento dedicó la sesión XIV, del 25 de noviembre de 1551 a la doctrina del sacramento de la penitencia. Durante el Concilio III Provincial Mexicano (1585), 
Por lo que hace a los franciscanos, los manuales para confesores que circulaban en la Nueva España trataban de orientar a los frailes en la comprensión de "las expresiones vernáculas apropiadas para los interrogatorios [del confesionario] [y para la] administración del sacramento de la penitencia". 9 Obras como el Confesionario mayor (1565) de fray Alonso de Molina guardaban ese sentido, lo cual pronto las situó entre los manuales religiosos de mayor uso en el ámbito novohispano. En cuanto a sus contenidos, textos como el Confesionario mayor recordaban a los sacerdotes la conveniencia de preguntar a los penitentes acerca de su relación con los curanderos y hechiceros, dado que con ello se esperaba corregir excesos y erradicar lo que llegaba a considerarse prácticas idolátricas. Las relaciones entre indios y españoles también eran motivo de indagaciones específicas, por lo que los interrogatorios en el confesionario se avocaban también a preguntar sobre posibles transgresiones a los 10 mandamientos en la vida diaria.

En tanto textos bilingües, algunos manuales para confesores han sido considerados fuentes invaluables para el estudio de las lenguas indígenas en la Nueva España. Además de ello, algunos estudiosos consideran que manuales como el de Molina ofrecen valiosos testimonios acerca de la situación de los grupos indígenas bajo el dominio español. Asimismo, estos textos han sido de ayuda para analizar tanto los contextos en que operaban los métodos de evangelización como la "mentalidad franciscana sobre los indígenas" y las formas de resistencia de éstos frente a la conquista espiritual. ${ }^{10}$

\section{CONTEXTOS DE ELABORACIÓN}

El Confesional en Carmeleño que aquí se transcribe forma parte de este género catequético que alcanzó una amplia difusión en el centro de la Nueva España, ${ }^{11}$ especialmente durante los siglos XVI y XVII, y que eventualmente

los obispos optaron por encargar la composición de un catecismo y manual para confesores aprobado por el propio concilio. Tejada y Ramiro (1859, pp. 633-636).

9 Molina (1984 [1565], p. 2).

10 Moreno (1984, p. 20); Murillo Gallegos (2010, p. XII).

11 Además del texto ya citado de fray Alonso de Molina, otros manuales para confesores que circularon en la Nueva España durante el periodo colonial fueron el Confesionario 
alcanzó la frontera norte en la medida que los misioneros se fueron instalando entre los grupos indígenas de esta amplia zona. Tal fue el caso de las misiones de la Alta California a partir del último tercio del siglo xviII, momento en que los franciscanos fueron convocados como parte del proyecto imperial por reforzar la presencia española en el noroeste novohispano. ${ }^{12}$

Dada la imposibilidad de precisar en este texto los pormenores del poblamiento misional y militar en la Alta California después de la expulsión de los jesuitas, vale la pena recordar únicamente que para 1768 la corona española tenía sobrados motivos para pensar que la frontera noroeste novohispana se veía amenazada por el avance de los rusos desde Alaska hacia el sur del litoral Pacífico, alentados por el comercio de pieles de nutria. En ese contexto, José de Gálvez dispuso que desde la península de Baja California, donde operaban los franciscanos del Colegio de San Fernando (en sustitución de los jesuitas expulsos), se organizaran expediciones para reconocer y poblar lo que eventualmente sería el territorio de la Alta California; así, para 1769 los primeros asentamientos españoles se establecerían en San Diego. Al año siguiente llegarían también más al norte, a la península de Monte Rey (también Monterrey

en lengua Mexicana y castellana (1599) y las Advertencias para los confesores de los naturales (1600) de fray Juan Bautista de Viseo; el Confesionario mayor y menor en lengua Mexicana y pláticas contra las supersticiones de idolatría que el día de boy an quedado a los naturales desta Nueva España (1634), de Bartolomé Alva Ixtlixóchitl; Luz y método de confesar idólatras (1692), de Diego Jaimes Ricardo Villavicencio; o el Arte, vocabulario y confessionario en el idioma mexicano, como se usa en el obispado de Guadalaxara (1765), de Gerónimo Thomás de Aquino Cortés. Entre los catecismos y las guías para la explicación de la doctrina cristiana fueron de especial relevancia la Doctrina Christiana (1546), de fray Alonso de Molina; el Catecismo de la doctrina Cristiana (1591) de Gerónimo de Ripalda (originalmente impreso en España y ampliamente conocido en la Nueva España durante la segunda mitad del siglo XVII); el Itinerario para párrocos de indios (1668), de Alonso de la Peña Montenegro; el Farol indiano y guía y cura de indios (1713), de fray Manuel Pérez; el Catecismo o Instrucción Christiana (1752), de Pedro Murillo Velarde; el Promptuario Manual Mexicano (1759), de Ignacio de Paredes; o el Catecismo para uso de los párrocos hecho por el IV Concilio Provincial Mexicano (1772). Para una lista más completa sobre los manuales impresos y utilizados en la Nueva España, véase Christensen (2013, pp. 51-95).

12 Hackel (2005, p. 3). 
o Monterrei, según las variantes de la época; para fines de consistencia, se adopta aquí el uso más común en castellano: Monterrey). En este último sitio, para 1770, fray Junípero Serra y sus compañeros establecerían la misión de San Carlos Borromeo, la cual sería trasladada unos meses más tarde hacia la ribera del río Carmel, a corta distancia del presidio de Monterrey. Debido a esta mudanza, la misión de San Carlos sería también conocida como Carmel, y los indios congregados en este sitio serían genéricamente identificados como Carmeleños. Hoy sabemos que al momento del contacto con los españoles en esta zona habitaban una gran diversidad de grupos étnicos, en su gran mayoría hablantes de la lengua Rumsen, una variante de la familia lingüística Costanoan. ${ }^{13}$

Desde la fundación de la misión de San Carlos, fray Junípero Serra y su compañero fray Juan Crespi encontraron en los niños de la bahía de Monterrey a los catecúmenos más constantes. Así, desde los primeros años de esta misión, Serra y Crespi contaron con la ayuda de un pequeño grupo de niños Rumsen que eran entrenados como intérpretes. Es muy probable que entre estos primeros catecúmenos se hubiera encontrado el intérprete que asistió a los fernandinos en la confección del Confesional en Carmeleño; el manuscrito carece de referencia explícita a su autoría, y hasta el momento sólo ha sido posible establecer algunas inferencias preliminares sobre la época de su redacción. ${ }^{14}$ Por lo menos dos indicios podrían servir para datar el Confesional en Carmeleño hacia la década de 1780. Por una parte, el ya conocido hecho de que una década más tarde, fray Fermín Francisco de Lasuén (continuador del trabajo misional en Carmel después de la muerte de Serra en 1784) compartiera con miembros de la expedición de Alejandro Malaspina (al momento de alcanzar la bahía de Monterey en septiembre de 1791) un vocabulario trilingüe en Castellano-Rumsen-Esselen. ${ }^{15}$ Por otra parte, la incorporación de la lengua Esselen en este tipo de textos para principios de la década de 1790 debe considerarse no solamente

13 Shipley (1978, pp. 80-90); Levy (1978, pp. 485-495); Milliken, Shoup, y Ortiz (2009, p. 19).

14 De la Torre Curiel (2017).

15 Alcalá Galiano (1802, p. 172); Cutter (1960, p. v). 
una expansión lingüística, sino un reflejo de los reacomodos demográficos de la misión de Carmel; como ha documentado recientemente Steve Hackel, para los últimos años del siglo XVIII este segundo grupo indígena constituía la porción más numerosa de los nuevos catecúmenos en San Carlos Borromeo. ${ }^{16}$

Finalmente, vale la pena señalar que la elaboración de este tipo de manuales para confesión fue una constante en las misiones franciscanas de California. Como han documentado autores como Maynard Geiger, Clement Meighan, James Sandos y Steve Hackel, el uso de este tipo de guías era más bien común para la última década del siglo XVIII en aquellas misiones, y por regla general centraban su interés en las prácticas sexuales y medicinales - descritas como "hechicerías" en dichos documentos- y en la recurrencia de los indios a "decir mentiras" a los padres. ${ }^{17}$ Sin embargo, el conocimiento actual que se tiene sobre estas actitudes en el confesionario, así como las evidencias indirectas sobre las prácticas locales indígenas, están basados en manuales preparados en otras lenguas nativas, probablemente con fecha de elaboración más tardía, y que carecen del detalle con que se describen y explican aspectos como el uso medicinal del tabaco, las sangrías, así como la actitud de frailes e indígenas frente al uso de los bienes de la misión y al relajamiento de reglas como el ayuno. ${ }^{18}$ Como en otro trabajo se ha señalado, la visión que este confesional ofrece sobre las alegadas transgresiones de los indígenas, el cuidado que se prescribe para tratar de erradicar alguna práctica medicinal —como el "chupar" o "ser chupado"19-, así como las recomendaciones que ahí se asientan para que el confesor reste importancia a "robar poco", o faltar a misa por

16 Hackel (2005, pp. 75-78).

17 Geiger y Meighan (1976, p. 71); Sandos (2004, pp. 97-120); Hackel (2005).

18 Véanse en especial las secciones "sobre el robar", "sobre la borrachera", y "sobre hechicerías" en la transcripción que se ofrece más adelante.

19 Se refiere a la práctica de colocar un objeto — piedra o madera— sobre algún área del cuerpo de quien se considera aquejado de un mal, para que después el curandero "chupe" ese objeto y retire el mal causante del desequilibrio. En la misión californiana de San Diego, un franciscano del siglo XVIII anotaba al respecto que cuando el curandero terminaba esta labor, se sacaba de la boca la pieza que había chupado y se le mostraba al enfermo, "y lo convence de que ese era el objeto que le causaba daño". Con ello, los 
alguna razón justificada, ayudan a tener una mejor comprensión de los contextos iniciales del establecimiento de las misiones en esta zona de la Nueva España. En otras palabras, los indicios de negociación y acomodo entre diferentes grupos de pobladores constituyen una nota por demás reveladora en este texto. ${ }^{20}$

\section{ESTRUCTURA Y LOCALIZACIÓN DEL DOCUMENTO}

$\mathrm{Al}$ igual que otros manuales para confesores de la época, el Confesional en Carmeleño ${ }^{21}$ presenta una serie de diálogos mediante los cuales el sacerdote debía proponer al penitente la conveniencia de alejarse de pecados específicos. De esta manera, la guía para la confesión constituye la sección principal de este documento mediante la siguiente estructura:

\begin{tabular}{|c|c|c|c|}
\hline SECCIÓN* & Título & CONTENIDOK' & FOLIO \\
\hline I & Sin título & [Advertencia / Dedicatoria en Latín] & ff. $1 v-2 f$ \\
\hline \multirow{6}{*}{ II } & \multirow{6}{*}{$\begin{array}{l}\text { Sin título } \\
\text { [Guía para la } \\
\text { confesión] }\end{array}$} & $\begin{array}{l}\text { "Al principio de la confesión" } \\
\text { [Invitación al penitente para confesarse] }\end{array}$ & f. $1 v-2 f$ \\
\hline & & $\begin{array}{l}\text { [Sobre el pecado de la } \\
\text { carne, las palabras y los } \\
\text { pensamientos deshonestos] }\end{array}$ & ff. $2 \mathrm{f}-4 \mathrm{v}$ \\
\hline & & "Sobre el robar" & f. $5 \mathrm{f}$ \\
\hline & & $\begin{array}{l}\text { "Sobre el aborrecer o } \\
\text { querer mal a otro" }\end{array}$ & ff. $5 \mathrm{f}-\mathrm{v}$ \\
\hline & & $\begin{array}{l}\text { "Sobre la borrachera } \\
\text { [alucinógenos y tabaco]" }\end{array}$ & ff. $5 v-6 f$ \\
\hline & & [Sobre asistencia a misa...] & f. $6 f$ \\
\hline
\end{tabular}

indios se tranquilizaban “juzgando que quedan libres del mal”. Geiger y Meighan (1976, p. 71).

20 De la Torre Curiel (2017).

21 Anónimo, Confesional en Carmeleño, sin lugar, sin fecha. Archivo Histórico Franciscano de Zapopan (Zapopan, Jalisco, México), Fondo Colegio de Guadalupe, Caja 53. 


\begin{tabular}{|c|c|c|c|}
\hline SECCIÓN* & TÍTULO & CONTENIDO**: & FOLIO \\
\hline \multirow{4}{*}{ III } & \multirow{4}{*}{$\begin{array}{l}\text { Sin título } \\
\text { [Acto de } \\
\text { Contrición. } \\
\text { Sección } \\
\text { intercalada en } \\
\text { el original] }\end{array}$} & $\begin{array}{l}\text { "Breve exhorto para mover a dolor } \\
\text { después de oída la confesión [y] antes } \\
\text { de la absolución" [Texto bilingüe] }\end{array}$ & ff. $6 v-7 f$ \\
\hline & & "Exhorto para la caridad y contrición" & f. $7 v$ \\
\hline & & $\begin{array}{l}\text { "Exhorto para la contrición y } \\
\text { caridad" [Versión en Carmeleño] }\end{array}$ & f. $8 \mathrm{f}$ \\
\hline & & $\begin{array}{l}\text { "Para excitar a la Esperanza" } \\
\text { [Texto bilingüe] }\end{array}$ & ff. $8 f-8 v$ \\
\hline \multirow{3}{*}{ IV } & \multirow{3}{*}{$\begin{array}{l}\text { Sin título } \\
\text { [Continuación } \\
\text { de la Guía } \\
\text { para la } \\
\text { confesión] }\end{array}$} & $\begin{array}{l}\text { Continuación [Sobre } \\
\text { asistencia a misa...] }\end{array}$ & f. $9 f$ \\
\hline & & "Sobre el no comer carne" & ff. $9 f-v$ \\
\hline & & "Sobre hechicerías" & ff. $9 v-10 v$ \\
\hline \multirow[b]{5}{*}{$\mathrm{v}$} & \multirow{4}{*}{$\begin{array}{c}\text { "Breve } \\
\text { explicación } \\
\text { de los otros } \\
\text { sacramentos. } \\
\text { En idioma } \\
\text { Carmeleño" }\end{array}$} & "Del Bautismo" & f. $11 \mathrm{f}$ \\
\hline & & "Sobre la Comunión" & ff. $11 v-12 f$ \\
\hline & & "Sobre el Matrimonio" & ff. $12 f-12 v$ \\
\hline & & $\begin{array}{l}\text { "Sobre el Sacramento de } \\
\text { la Santa Unción" }\end{array}$ & ff. $12 v-13 f$ \\
\hline & $\begin{array}{l}\text { "Para la } \\
\text { aplicación de } \\
\text { la Indulgencia } \\
\text { in articulo } \\
\text { mortis, y } \\
\text { explicarles } \\
\text { su valor en } \\
\text { cuanto se } \\
\text { puede" } \\
\text { [Texto } \\
\text { bilingüe] }\end{array}$ & [Absolución en artículo de muerte] & $\begin{array}{l}\text { ff. } \\
13 v-14 f .\end{array}$ \\
\hline
\end{tabular}

Fuente: Anónimo, Confesional en Carmeleño. Archivo Histórico Franciscano de Zapopan (Zapopan, Jalisco, México), Fondo Colegio de Guadalupe, Caja 53.

* El manuscrito no incluye números de secciones, ni presenta esta división de manera explícita. Estos añadidos se proponen en beneficio de una exposición más clara.

** Los títulos entre comillas sí aparecen en el original; se agregan algunos títulos entre corchetes para tener una mejor visión del conjunto. 
El manuscrito es un cuadernillo de 14 fojas, con medidas aproximadas de $17.5 \mathrm{~cm}$ de largo por siete $\mathrm{cm}$ de ancho. En la actualidad se conserva en el Archivo Histórico Franciscano de Zapopan, lugar a donde se trasladó el archivo del antiguo Colegio de Propaganda Fide de Guadalupe Zacatecas. Es probable que el manuscrito hubiera llegado a Zacatecas entre las décadas de 1830 y 1840, cuando diversos religiosos de este convento fueron enviados a California para ayudar en el cuidado de aquellas misiones ante la incapacidad del Colegio de San Fernando de México para seguir enviando religiosos a esos destinos.

\section{CRITERIOS DE TRANSCRIPCIÓN}

Con el fin de permitir la lectura que ofrece la estructura del texto original, en esta edición se optó por presentar un texto a dos columnas, de manera que el ejercicio de lectura resultante replicara la experiencia de contrastar en un mismo momento el contenido del verso (columna izquierda) y el frente (columna derecha) de cada par de páginas del Confesional.

Asimismo, se sigue la práctica de modernizar la ortografía y desdoblar las abreviaturas (que, cuando, cuanto, para, pero), conservando, en cambio, algunos términos y conjugaciones verbales presentes en el original con el fin de mantener el tono que ofrece el documento original. En este sentido, se optó por la homogeneización en el uso de "v" e " $y$ " en aquellas palabras cuya escritura ofrecía variantes con " $b$ " o con "i"; se acentuaron los verbos en pasado, y se sustituyó la "z" por "c" en varios verbos en pasado.

En la estructura de párrafos y columnas se respetó la separación de líneas que emplea el documento original, y se homogeneizó el uso de guiones para los cortes de palabras entre líneas.

El texto original presenta los diálogos del interrogatorio en pequeños bloques que en la transcripción quedan marcados mediante el empleo de llaves “ \{\} ” para conservar la idea de unidad dentro del interrogatorio. De igual forma, se conservó el énfasis en aquellas palabras que aparecen subrayadas en el documento y se respeta el uso del original de trazar separaciones entre bloques específicos mediante el uso de líneas punteadas.

Finalmente, con la intención de destacar visualmente las variantes entre castellano y rumsen, se decidió emplear cursivas para el texto en rumsen, 
conservando en redondas los préstamos lingüísticos empleados por los misioneros para expresar en esta última lengua algunos conceptos básicos del cristianismo.

\section{CONFESIONAL EN CARMELEÑO}

\section{[f. 1v]}

Adviértase que la $\underline{X}$ en lengua tiene fuerza como de $\mathrm{C}$ y J (algo mas fuerte).

Al principio de la Confesión[:]

No tengas miedo, no tengas vergüenza para confesarte. No te regañaré. Por eso confiesa todos los pecados que hiciste, para que Dios te perdone....

\section{[f. 2f]}

$+$

Te Domine qu[a]esumus, ut ex ore nostro incesanter tibi laudes perficias, qui Beato Gentili. Martiri tuo ad Infidelium conversionem linguarum donum Indidisti: Per Dom.

Al principio de la confesión[:]

\{Cue cume xojolon, cue cume uchan ezcume confesar. Cue cu ca mes huiai. Nepsoma oqueija inmei nume xincei mur gici quechens, ezcumes Dios aanan....

Comenzando por el pecado mas conocido de ellos mismos entre los mas comunes....

\{Mutz mure attai lachianc?

[¿]Has robado mujer?\}

Pregunta para el hombre 
\{Mutz mure attai muquianc?

Has - robado hombre[?]\}

Pregunta para la mujer: así enti-

enden bien, aun resp[ec]to de la mujer, el pecado de la carne.

Sina lachianc ${ }^{22}$ (muquianc $)^{23}$

num rexincei attai

mutz urrin ass soltera.

Esa mujer (hombre) que

robaste [i]era casada o sol-

tera [?] [f. 2v]

\{Muts urrin.

[¿]Es casada?\}

Pero para preguntar a la mujer que pecó con el hombre debe preguntársele

\{Muts jaunant?

[i]Es casado[?]\}

$\{$ Muts mure attai antiguis

lachianc?

[¿]Has robado a otra mujer?\}

\{Muts mure attai antiguis

muquianc?

[¿]Has robado a otro hombre [?]

Esto es [i] has pecado con otro hombre?\}

Para preguntar por ejemplo

cuántas veces ha pecado[:]

\{Imjame atsap?

[¿]Cuántas veces?\}

22 En la parte superior del texto, sobre "lachianc" se indica: "si es mujer".

23 En la parte superior del texto, sobre "muquianc" se indica: "hombre". 
\{Cue cume guatin richaon nusina lachianc numure xincei atthai No vayas [a] hablar a esa mujer que robaste antes\}

Si en lugar de nussina lachianc se dice nussina muquianc, con lo demás; quiere decir no vayas a hablar a ese hombre \&.... [f. 3f]

\{Cue cume guatin aieu nusina lachianc numure xin cei attai No vayas a verle a aquella mujer que antes robaste\} Si se dice a la mujer que no vea al hombre: en lugar de lachianc se dice $m u$ quianc

\{urri cume nusina muquianc, numure xincei atthai Aparta o echa a aquel hombre con quien pecaste\}

\{urri cume nusina lachianc

Echarás a aquella mujer \&\}

Si es conv[enien]te preguntar de ocasión próxima \{Muts an inmensa rucat, numure xincei gici quechens?

[¿]Vives juntamente con aquel con quien hiciste cosa mala?\} 
[f. 3v]

Qeet cume guas

Échale afuera.

\{Qeet cume guas numure xincei gici quechens

Échale afuera a aquel

con quien pecaste\}

\{Imats guat me ruc

qeet cume guas

Si viene a tu casa, échale.\}

En lugar de Qeet cume guas

se puede decir jurri cume guas.

Pero el Qeet cume guas pare-

ce mas propio

\{Muts mure richiiquechensi

richass?

[i]Has hablado malas pa-

labras?\}

Este richii quechensi richas

quiere decir hablar malas pa-

labras, en general; sean ma-

las por deshonestas, o ya

sean malas por nombrar

al Diablo o a raicos como dicen.
El propio de hablar deshonesto

de cosa de la carne es xamiarp

$\{$ Mutz mure xamiarp lachianc? [i] Has hablado deshonestidades de cosas de mujeres?\}

\{Mutz mure xamiarp muquianc?

[i] Has hablado deshonestidades de hombres?\} 
I64 JOSÉ REFUGIO DE LA TORRE CURIEL · EL CONFESIONAL EN CARMELEÑO

[f. 4f]

\{Cue cume atzap xamiarp.

Para preguntar si consen-

No hables mas deshonestidad[e]s\}

tía[:]

\{Imatz muquianc, imatz la-

\{Tanamur ez guat nesina

chianc uti xamiarp.

quechensi pesoi, mutz mure

Cue cume raguanp.

Cue cume siuniin tanai

jurri tanai sina pesoi que-

chens?

juia me sirre.

Cuando te vinieron estos

Cuando los hombres, o cuando malos pensamientos [i] echaste las mujeres hablan deshoentonces aquellos pensam[iento]s nestidades: no respondas, malos?\}

no te alegres entonces en

tu corazón.\}

\{Cue cume igest xamiarp.

No sepas hablar desvergüen-

zas, o deshonestidades\}

$\{$ Mutz mure pesoi quechens

lachianc?

[¿]H Has pensado cosas malas de

mujeres?\} Si en lugar de $\underline{l a-}$

chianc se dice muquianc

es de mujeres (hombres) ${ }^{24}$. Atsianc de

muchachas: $\underline{\text { Sinianc de }}$

muchachos: según uno qui-

era preguntar.

24 En el original aparece tachado "mujeres". 
[f. $4 \mathrm{v}$ ]

\{Imatzez guat quechensi pesoi

\{Cue cume atzap tatagni me

lachianc jurri cume utiesin

nesina pesoi quechens.

Cuando te vengan pensam[iento]s

turrum juia me huinum

imatzez guat quechensi

malos

de mujeres muquianc

de hombres, echarás los

pesoi.

No te toques tu cuerpo abajo quando te vienen malos

estos pensam[iento]s malos.\} pensamientos.\}

\{Imatzez guat sina pesoi

quechens lachianc cai cu-

me tanai Jesus María.

Cuando te vengan aquellos

malos [pensamientos] de mujeres

muquianc

dirás entonces Цesús Maria.\}

\{Tanamure guat quechensi pe-

soi lachianc, mutz mure

tatagni me turrum juia

me huinum?

Cuando te han venido ma-

los pensam[iento]s de mujeres $\underline{m u-}$

quianc si es de hombres,

[i] te has tocado tu cuerpo en

tu abajo?\}

Con esto entienden los ma-

los tocamientos 
[f. 5f]

$\underline{\text { Sobre el robar }}$

\{Mutz mure attai intaseia?

[¿]Has robado alg[u]na cosa?\}

Trigo, cebado, ijol, por frijol,

las otras comunes de voces

castellanas como maíz,

chícharo etc..en la misma

Castilla entienden comun-

mente.

\{Anitie mur attai? [¿]Donde robaste?

Mutz mure athai Mis[io]n, he-

ra, milpa, etc.

[¿]Robaste a la Mis[ió]n, en la era

o milpa?\}

$\{$ Mutz mure ege attai

ax puciut

[¿]Robaste mucho, o poco [?]\}

\{Imats $\hat{a}$ attai puciut

cue sina ege quechens

Cuando se roba poco no es

mucho pecado.

\{Cue cume ege attai.

No robes mucho.\} $\underline{\text { Sobre el aborrecer o querer mal a otro }}$

\{Mutz mure amai chajsun?

[i]Has estado aborreciendo a

alguien?\}

\{Cue cueme uas chajsun

No le aborrezcas.\}

$\{$ Mutze uas richavon?

[¿]Le hablas?\}

\{Richavon cume vas misijai sirreion? [¿]Le hablabas con buen corazon[?]\} 
[f. 5v]

Mutz mure amai zit?

[¿]Te peleaste con alguien?

Mutz am posc siuniinin?

[¿]Os contentásteis?

A la prim[er]a pregunta que se ha hecho sobre este 5 mandam[ien]to de no querer mal a otro; se podrá añadir:

\{Tanamure uas chajsum mutze uas post iusicin?

De cuando le aborreciste

[i] te has puesto después a quererle bien?\}

Si da a entender que no dígasele \{Nei cume uas iusss Desde ahora quiérele bien.\}

Si a la pregunta que se le hizo

de [i]os contentasteis? dice que no[,] dígasele

\{Qechuez co am siuniin

Te contentarás con el cuanto antes\}

\{También Quechuez co am richamo.

Te pondrás a hablar cuanto antes
Sobre la borrachera

$\{$ Mutz e igest maziann?

[¿]Eres borracho?\}

\{Tanamure amjai zaguans.

Mutz mure mazian, mutz

mure tonei me sirre zagu-

anizon?

Quando comiste tabaco

[i] te emborrachaste o te

perdiste tu cabeza con tabaco?\} 


\section{[f. 6f]}

Si dice que se perdió su cabeza con tabaco

$\{$ Muts mure momei?

Quiere decir si estuvo mucho o le duró mucho aquella borrachera?\}

\{Tanamure amjai zaguans, mutz mure mazzian tanamure zorrou?

Quiere decir: si se emborrachó bebiendo la bebida que tienen algunos de Tabaco compuesto con cal?\}

\{Cue cume am zaguans zorrouon: ezcume cuetonei me sirre. No bebas lo del tabaco con cal; para que no te pierdas tu sentido:\} \{imatse uques, picit co $\hat{a}$, picit co â: si bebes, sea poquito.) (pero hágase por desterrar este uso) sea poquito.

\{Cue cume am zaguans egien imatse am zaguans, pictit co a No comas tabaco mucho. Si comes tabaco sea poco.\}

$\{$ Ez cume cue tonei me sirre.

Para que no te pierdas tu sentido.\}
$\{$ Mutz mure jurriri Misa tanamur Domingo, tanamur fiesta? [i]Has dejado [de ir a] misa cuando era domingo[, o] cuando era fiesta? Si dice que si\}

$\{$ Mutz mure tanai inat? [¿]Estabas tu entonces enfermo? Si dice que si, se le dice\}

\{Imano jurrin Misa imano inatjim cue sina quechens. Cuando se deja la misa estando enfermo no es pecado\} 
[f. 6v]

Lo que falta del interrogatorio del examen

está después de las dos páginas inmediatas $^{25}$

Traduccion literal a la Castilla

Cuando te fuiste a pecar el diablo te engañó, para llevarte al infierno: Cuando hiciste pec[ado]s el Diablo entró a tu alma: si vieras a tu alma fea como está cuando hiciste pecados, te morirías de susto: El diablo estaba allí; allí en tu Alma quando hiciste pecados, cuando ofendiste a Dios: el Diablo te movía tu alma, cuando tu hacías pecados, cuando pensabas cosas malas, el Diablo te movía tu Alma para llevarte Al Infierno.

Mira... por esos pecados que hiciste te estaba para echarte Dios al Infierno: allí en el Inf[ier]no te abrasarías como un leño se abrasa: te abrasarías siempre a todo tiempo, todo el dia, de noche, de medio dia, de maña- na, de tarde, siempre, siempre te quemarías como un leño que se quema, te atormentarías; por esto siéntete de tu corazón de haber pecado; llora en tu corazón, que el Demonio te engañó para llevarte al Infierno.

[¿]Quisieras no haber tu hecho pecados? Cue: [Este cue por respuesta a aquella preg[un]ta quiere decir: Quisiera, no haber pecado. O lo que es lo mismo; No, no quisiera haber pecado que por lo anterior incluye acto de Atrición:]

\section{$[\dot{e}]$ Amas tu a Dios?}

25 Continúa en la foja 9f. 
Breve exorto para mover a dolor después de oida la confes[ió]n antes de la absolucion. En Carmeleño.

Tanamure guatín gici quechens yechen mes iolecin ezcumes guatis huinun, Tanamurne gici quechens, yechen aconsin mesirre; imatone aieu me sirre quechenis juia rotei tanamure gici quechens lacui ton mexojolon: yechen juia tauracin juia me sirre tanamure gici quechens; tanamure e tarmanp Dios: yechen mure ginevon me sirre tanamure gici quechens, tanamure pesoi quechens, yechen murez ipe me sirre ezcumes guatín huinum....

Cume xac: Neme xincei giciecin quechens, oquepach murez Dios buinun: nu juia buinum zurren ton cume catano tizii zurren; $\mathrm{Zu}$ rren ton cume um aipirre, aituugs, orpeteu, tugeieu, changuai, buiacau, iim, iim, zurrenton cume catano tizii zurren, huijait ton cume. nepsoma churren cume sirre me giciecin quechens; huare me sirre, yechen mes iolecin ez cumes guatis huinum.

Mutz tunumure cue iusen g[ici] quechens? Cue.

Para el acto de contric[ió]n se puede comenzar asi.

Mutz e muicin Dios? 
[f. $7 \mathrm{v}$ ]

Exhorto para la Carid[a]d y

Contric[ió]n

Dios te ha dado todo cuanto tienes; Dios te dio Alma y Cuerpo, Dios te ha hecho para llevarte al Cielo, para que tengas todo contento en el Cielo por eso Dios te hizo.

El mismo Dios se hizo hombre, para llevarte al Cielo: el mismo Dios que se hizo hombre se llama J[esu] C[ris]to.

Este J[esu]C[ris]to es Dios, y también hombre.

El mismo J[esu]C[ris]to murió crucificado en la S[an]ta Cruz para llevarte a los Cielos. Si no hubiera muerto J[esu]C[ris] to

en la S[an]ta Cruz, a todos nos hubiera [llevado] el Diablo al Infierno: por eso quiso morir JesuC[ris]to, quiso padecer JesuC[ris]to para llevarte a los Cielos; para que tu tengas todo contento en los Cielos, por esto murió crucificado en la S[an]ta Cruz J[esu]C[ris]to: por esto, mucho te ama Dios como hijo suyo, por esto amarás tu también mucho a Dios. Todo bueno Dios, mucho bueno Dios. Todo Grande Dios: todo bueno Dios. Por esto, ¿le amas a Dios? Responde Si.

Pero cuando hiciste pecados[,] cuando pensaste malos pensam[iento] $\mathrm{s}[$, cuando hiciste pecados le querías tu al demon[i]o, no le querías a Dios. Cuando hiciste pecados querías lo que manda el Dem[oni]o: enojabas a Dios.

Por eso, [¿]quisieras no haber pecado? Si responde cue. Ya está... 
[f. $8 \mathrm{f}]$

Exhorto para la Contrición y Caridad Inmeia misig Dios, ege misig Dios. Inmeia Isacai Dios, inmeia misig Dios.

Dios mes arracin nume nemexincei anmis, Dios mes arracin sirre turrum, Dios mes giciecin ezcumes guatis Nepsoma mutze muicin Dios?

Responde

Si.

tarrajta, ez cume anmis inmei siunim juia tarrajta nepsoma Dios mes giciecin:

Huacai Dios hua giciepcin muquianc ez cumes guatis tarrajta: huacara Dios huagiciepcin muguianc caigina ract JesuC[ris]to: Nesina J[esu]C[ris]to Dios, iss muquianc.

Huacaîa][esu]C[ris]tohualacunsinhuapacastap juia S[an]ta Cruz ez cumes guatis tarrajta. Imaton mur cue lacun J[esu] C[ris]to juia S[an]ta Cruz, inmei tunumur guatis maquis yechen huinun: nepsoma

hua incencin lacun murez J[esu]C[ris] Mutzeianis neia cumesto

bua incencin huijait murez J[esu]C[ris] to

ez cumes guatis tarrita; ez cume anmis inmei siuniin juia tarrajta nepsoma bua lacunsin huapacastap juia S[an]ta Cruz: nepsoma ege mes muicin Dios cata huacai Insins, nepsoma muicin quex cume ege Dios.

Eneha Tanamure gici quechens tanamure pesoi quechens, tanamure gici quechens muicin man mure yechemin, cue man mure muicin Dios; tanamure gici quechens unn man mure nip. Yechen, tanmanp man mure Dios.

Nepsoma. Mutz tunumure cue iusen gici quechens? Responde Cue...

Para excitar a la esperan-

za. 
[f. 8v]

anan Dios inmei me quechens? Luego los Actos de Fe, Espe-

Tanamura lacunsin JesuC[ris]to ranza y Caridad, como

Juia Santa Cruz, JesuC[ris]to bua están en lengua.

iispicim mac quechens; hua-

cai Dios misig, inmeia tu-

man Dios, huacai rutacim,

ezcu maquis anan quechens

imassac confesar, nep-

soma mutze ianis neia

cumes anan Dios inmei

me quechens?

Et.

Quiere decir esto desde el prin-

cipio de este superior párrafo

comenzando desde el mutze ianis [...]?

[¿]Esperas que ahora te perdonará

Dios todos tus pecados?

Cuando murió JesuC[ris]to en la

Santa Cruz, JesuC[ris]to pagó tus

pecados, el mismo Dios bueno,

el todo poderoso Dios, el mis-

mo tiene dicho, que nos perdo-

nará los pecados cuando

nos confesamos; [ं]

peras que te perdonará aho-

ra Dios todos tus pecados?

Si. 
[f. 9f]

$\{$ Mutz mure jurrin Misa tana-

mur me misig?

mas claro:

[i]Has dejado Misa estando bueno? $\}^{26}$

$\{$ Cue quechens

imano tanai amajam tozze

\{Imjame mur jurrin Misa

imano inatjim.

tanamure misig, me unionsin.

No es pecado cuando se come

[¿]Cuántas veces dejaste Misa cuando

la carne estando enfermo\}

estabas bueno, por tu flojera?

Sobre el no comer carne

\{Tanamur cue me inat tanamure misig, mutz mure amjai tozze tanamur Viernes

\{Mutz mure amjai tozze, tanaQuaresma?

mur cai Padre cue cuman Cuando no estabas enfermo, amjai? Tanamur Viernes Cuaresma? cuando estabas bueno; [c] comis-

[¿]Has comido carne quando te carne en Viernes de cuaresma?\}

el Padre dijo que no comeréis?

[¿]Cuando era Viernes de cuaresma?\}

Si dice que si, se preg[un]ta luego[:]

Si dice que si:

\{Mutz mure tanai inat?

[¿]estabas entonces enfermo?\}

Si dice que si[:]

\{Imatse amjai tozze imatse

inat, cuesina quechens.

Si comes carne si estás en-

fermo no es pecado.

26 Con esta línea se retoma el cuestionario de la foja $6 f$. 
[f. 9v]

\{Mutach mure ani pesoi tanai

En lo expuesto parece

tuugs tanamur cai Padre

cue cumam am tozze?

estar comprendido un

[i]Te acordabas que era aquel

interrogatorio general

para los indios en común.

día del que había dicho el

Padre que no comiérais carne?\}

Uit cume sina yenepin, sina chochon...

Si dice por último que si, luego[:]

Quitarás esas cosas etc. ...

IImjame mur amjai tozze?

[¿]Cuántas veces has comido carne?\}

Sobre hechicerías

$\{$ Mutz mure ogzcien?

[i]Has hecho hechicerías?

Cue cume ogzcien.

No hagas hechicerías.\}

\{Yechen juia aq juia ojzcien

nepsoma cue cume ogz-

cien.

El demonio se mete en

las hechicerías: por eso no

hagas hechicerías.\} 


\section{[f. 10f]}

Si pareciere conv[enien]te el sondear sobre las hechicerías o supersticiones. Pregúntese[:]

\section{\{Mutz mure yenepigni?}

Esto quiere decir:

si ha puesto semilla u otra cosa en algún lugar como ofreciendo al Diablo:\}

Todo lo dicho quiere dar a Entender el yenepigni

\section{\{Mutze a is rotei yenepin?}

[i]Tienes todavía puesta alguna cosa como semilla $\&$ en algún lugar en ofrecimiento al Diablo?\}
El chochont significa el palo semejante.

\{Uitp cume sina yenepin, sina chochon.

Quita esas cosas etc.\}

$\{$ Mutz mure amai loj?

[¿]Has chupado a alguno?

\{Mutz mure lojojon?

[¿]Te han chupado?

De modo que el loj es chupar, activo. El lojojon es pasivo: ser chupado [ilegible]

\section{[Cue cume atzap yenep.}

No hagas mas tales ofrendas\}

\{Mutp cume sina yenepin?

Quemarás esa cosa de ofrenda.\}

Uitp cume sina me yenepin.

Tíralo, esa hechicería.

\{Mutz mure chochont?

[¿]Tienes puesto algún palo donde pongas alguna cosa como ofreciendo al Demonio?\} 


\section{[f. 10v]}

Si dice que ha chupado, o que le han

[Cue cume atzap loj.

chupado otros, se podrá preguntar. No chupes mas.\}

Mutz mure iusen tanai yechen

ez mure ez zai?

$[\dot{i}]$ Has querido entonces que el Diablo te ayudase?\}

Y lo mismo se podrá preguntar cuando dice las supersticiones anteriores.

\section{\{Tanamure lojojon mutz mura}

nom jechen.

[¿]Cuando te chupaban llamaban

al Diablo?\}

Si dice que si se preguntará lo que antes.

$\{$ Mutz mure iusen tanai yechen

ez mur ez zai?

[¿]Has querido entonces

que el Diablo te ayudase?\}

\{Mutz mure mai tanai

yechen ez murez zai?

[¿]Has creído que el Diablo

te ayudase?

\section{¿Cue cume atzap lojojon.}

No te dejes mas chupar.

Pero si no hay en lo dicho cosa alguna de superstición no hay por qué mandar lo anterior 


\section{[f. 11f]}

\section{$\left\{\right.$ Mutz mure igest amai îirree? ${ }^{27}$}

[¿]Has hecho superstición en curar

[a] alguno?\}

El verbo ûirree ${ }^{28}$ parece que abraza

la superstición que tienen al t[iem]po de curar a algún enfermo.

La superstición esta en que chupando, o sea con baile que hacen para curarle piensan impedir al Diablo que les haga mal.

\section{[Mutz mure igestigin virregen[?]}

[¿]Te han hecho la dicha superstición cuando te han curado?\}

El virree es activo: esto es significa la superstición activa de ellos en dichas diabólicas curaciones.

El virregen es pasivo, y significa dejarse curar de dicho modo.
Se les puede decir luego lo que sigue:

[Cue cume maai imase amai loj. No creas, que si chupas a alguno,\}

\section{Limasse amai virree}

que si haces la dicha superstición,\}

\{neme sina yechen zaz

que con esto te ayudará el Diablo.

\{Ene cume cue amai nei loj.

Así[,] no chupes mas ahora a alguno,\}

\{cue cume, cue amai virre. no hagas la dicha superstición.\}

27 En el original, la palabra ûirree está colocada sobre otra palabra tachada: vizree 28 En el original, la palabra ûirree está colocada sobre otra palabra tachada: vizree 
[f. 11v]

ene imatse amai ual

pero si solo sajas a alguno no mas,

oss cue cu neia quechens

Si que no es esto pecado

imatzan cue juia gineuon yeche-

min.

si no queréis en esto traer al Di-

ablo. verbo ûal que es cortar o sa-

jar, tiene buen sentido, y que solo sacan sangre sin superstición como se ha dicho: pero el $\underline{l} j$ que es chupar, o el uirrèè que parece aun peor, dan a significar la ya mencionada superstición.

En lo dicho se les advierte que el sajar solo para sacar sangre al enfermo no es malo, como no quieran que intervenga en ello el Diablo, al mismo t[iem]po que se les dijo primero que no chupen mas ni que hagan con los enfermos lo que ellos llaman ûirrèè, que viene a ser el bailar o otra cosa semenjante para llamar al Diablo, o tal vez para ahuientarle. De modo que el 
[f. 12f]

Breve explicación de los otros

Sacram[en]tos en idioma Carm[ele]ño

Del Bautismo

\section{Imatse humbriti, imatse bautizar}

Cuando, cuando te bautizas,

\{nei cumes anan Dios immei quechens te perdona Dios entonces tus pecados\}

\{Imatse bautizar, imase humbriti.

Cuando te bautizan, cuando te lavan en baut[iz]o\}

\section{\{Insinis tanai cume Dios}

hijo serás entonces de Dios.\}

\{Ianis cume inmei nesina

Espera todo esto.

\{Ianis cume anan cumes Dios

Espera que te perdonará Dios\}

Inme me quechens imatse bautizar todos tus pecados cuando te bautizan,

\{Incat hua lacunsin J[esu]C[ris]to aqueson

porque murió J[esu]C[ris]to por nosotros [f. 12v]

De la confesión ya se dijo antes

Sobre la comunión lo siguiente

De la comunión.

Mai cume.

has de creer

imano mis, juia hostia, que cuando la misa, en la hostia,

imano Padre nusincei richii juia hostia, juia ev JesuC[ris]to cuando el Padre dice las palabras en la hostia, allí viene JesuC[ris]to.

$\{$ Los dos renglones superiores son interpretación de los dos anteriores\}

Nepsoma imano Mis, imano

Padre nuxincei richiii, juia hostia, juia eu JesuC[ris]to por eso cuando la misa, cuando el Padre dice palabras en la hostia, allí viene JesuC[ris]to.

nepsoma imano ama Comulgar nuxincei och Padre amai jaitac huacaia JesuC[ris]to Dios, is muquianc.

por eso cuando comulga la gen- 


\section{[f. 13f]}

te, aquello que pone el Padre a la gente en la boca es el mismo J[esu] Chr[ist]o

Dios y hombre.

$E v \mathrm{~J}[\mathrm{esu}] \mathrm{Cr}[$ ist $]$ o mac sirre, imano mac comulgar, J[esu]Chr[ist]o muiemp mac sirre, immano mac comulgar.

Viene J[esu]Chr[ist]o a n[uest]ros corazones, cuando comulgamos J[esu]Chr[ist]o mantiene n[uest]ras Almas, cuando nosotros comulgamos. gar.
Repítanse los últimos 3 renglones

$E u$ JesuCr[ist]o mac sirrè, imano mac comulgar, JesuChr[ist]o muiemp. mac sirre, immano mac comul-

Viene JesuChr[ist]o a nuestros corazones cuando comulgamos, J[esu]Chr[ist]o mantiene o alimenta $\mathrm{n}$ [uestr]as almas, cuando nosotros comulgamos.

Huacai Dios, huacai mur mons inmei nesina; cue maquis igest iol Dios, cue tuman maqis iol Dios, nepsoma mai cume sirreion nepes. El mismo Dios, el mismo ha dicho estas cosas; no nos engaña Dios[,] no puede engañarnos Dios, por eso creerás de corazón esto.

Sobre el matrimonio: qué se ha de advertir cuando se van a casar y también cuando convenga a los que están casados para advertirles sus obligaciones

Del matrim[oni]o: Imano Chrisitiano, Imano exegts jaugüespojon, huacai Dios amai arra vq. 
Cuando los Christianos, Cuando los Christ[iano]s se casan, el mismo Dios les da compañero.

xinxoma Christianos exegts xinxoma isti Jangüespojon ez tei uti, insins ez uti guatin tarrajta.

Por eso los Christianos, por eso se casan, para tener hijos, para que vayan a los cielos.

\section{[f. 13v]}

Mai cu macam, neman xincei

gici ne, imano jauguespojon, $\mathrm{S}$ [an]to Sacramento Matrimonio, huacai amai nipasin JesuC[ris]to nepes.

Habéis de creer, esto que hacéis, cuando se casa; es S[an]to Sacramento del Matrimonio; el mismo J[esu] Chr[ist $]$ o enseñó esto.

Nepsoma hua urrin muicin co Jaunan, hua Jaguan muicin co urrin:

El marido ha de querer a la mujer, la mujer ha de querer al marido.

Hua Jaguan maicu uti urrin, bua urrin cue cu jurri jaunant: hua Jaguan quex cue cu jurri urrin.

La mujer ha de obedecer al marido; El Marido no debe largar a la mujer: la mujer tampoco debe largar al marido. 


\section{[f. 14f]}

Sobre el Sacram[en]to de la S[an]ta Unción

Nemes sina huat Padre arras

Santo Sacramento Extrema Unción:

huacai giciecin JesuChr[ist]o, huacai amai nipasin JesuChr[ist]o.

Esto que te viene el Padre a darte, es Santo Sacram[en]to de la Extrema Unción

Nemes sina buat Padre arras

$S$ [an]to Sacramento, ez comes Dios

arra

geech, ezcume rettepo neme sina

hiim caai, ez cume cutap

misijai sirreion.

Esto que viene [el] Padre a darte, es S[an]to Sacram[en]to; para que Dios te de [el] mismo JesuChr[ist]o instituyó, el mismo J[esu]Chr[ist]o lo enseñó. fuerza, para que sufras eso que te da dolor, para que lo lleves con buen corazón:

$\{$ Ez cu ûguin yechen; $e z$ cu quex Dios ames anan quechens nepsoma nesina Sacramento S[an]ta Uncion, nemes sina huat Padre arras.\}

\{Para vencer al Diablo; para que también te perdone Dios los pecados, por esto es este Sacra$\mathrm{m}$ [en]to de la Santa Uncion, que te viene [a] darte el Padre.\}

Ianis cu me inmei nesina arra cumes Dios inmei nesina incata huacaia JesuC[ris]to hua lacunsin juia S[an]ta Cruz hua pacastap J[esu]C[ris]to maqueson. 
Espera todo esto[,] te dará Dios todo esto, porque el mismo JesuC[ris]to murió en la S[an]ta Cruz y fue crucificado JesuChr[ist]o por nosotros.

\section{[f. 14v]}

Para la aplicación de la Indulg[enci]a in articulo mortis, y explicarles su valor en cuanto se puede.

Para la Indulgencia.

Cuttap cume a ne-

me xincei hiim caai, neme xincei lacuet misijai sinrreion, Dios iusen neme xincei lacut, nepson cuttap cume a misijai sirreion, ez cume iisp me quchens. Imats Dios iusen lacui cume cuttap cume a misijai sirreion, nepson cumes anan Dios nuxincei richii Padre nûch mure ez iispme quechenis anni pirre juia Purgatorio.

Para excitar a amor de Dios y dolor de los pecados véase donde se trata de la confesión. 
[f. 15f]

Explicacion en Castilla de lo que se pone en lengua para la aplicación de la S[an]ta Indulg[enci]a.
Para excitar a dolor de los pecados, y actos de amor de Dios sirve lo qu está puesto antes para la Confesión.

Para la Ind[ulgenci]a.

Sufre eso que

te da dolor, ese mal

que tienes con buen Corazón, Dios

quiere ese mal que tienes, así

pues[,] lleva con buen Corazón

para que pagues tus pecados.

Si Dios quiere que te mueras,

llevarás con buen Corazón,

por eso te perdonará Dios

con las palabras del Padre, lo que debías pagar por tus

pecados en la otra vida en

el Purgatorio.... 


\section{REFERENCIAS BIBLIOGRÁFICAS}

- Alcalá Galiano, D. (1802). Relación del viage hecho por las goletas "Sutil" y "Mexicana" en el año 1792 para reconocer el estrecho de Fuca. Madrid: En la Imprenta Real de José Espinosa y Tello.

- Aspell, M. (2008). El sacramento de la confesión. Los manuales y las sumas de confesión, Anuario del CIJS, 11, 431-447.

- Christensen, M. Z. (2013). Nahua and Maya Catholicisms. Texts and Religion in Colonial Central Mexico and Yucatan. Berkeley: The Academy of American Franciscan History, Stanford University Press.

- Cutter, D. (1960). Malaspina in California. San Francisco: John Howell Books.

- De la Torre Curiel, J. R. (2017). Within the Confessional: Franciscan Utopias and Daily Practices in Mission San Carlos. En Hackel, S. (coord.) Junípero Serra: Context and Representation, 1713-2013. Berkeley: University of California Press.

- De los Reyes Gómez, F. (2004). El Modus Confitendi y Andrés de Escobar. En Escobar, A. Modus Confitendi. Manual para la confesión (pp. 24-71) Burgos: Fundación Instituto Castellano y Leonés de la Lengua.

- Denzinger, H. y Hünermann, P. (1999). El magisterio de la Iglesia. Enchiridion symbolorum definitionum et declarationu, de rebus fidei et morum. Barcelona: Herder.

- Geiger, M., y Clement W. M. (1976) As the padres saw them: California Indian life and customs as reported by the Franciscan missionaries, 1813-1815 (editores y traductores). Santa Barbara: Santa Barbara Mission Archive Library.

- Hackel, S. (2005). Children of Coyote, Missionaries of Saint Francis: Indian-Spanish relations in Colonial California, 1769-1850. Chapel Hill: University of North Carolina Press.

- Levy, R. (1978). Costanoan. En Sturtevan, W. C. (editor). Handbook of North American Indians, vol. 8 (pp. 485-495). Washington D. C.: The Smithsonian Institution.

- McLaughlin, R. E. (2008). Truth, Tradition, and History: The Historiography of High/Late Medieval and Early Modern Penance. En Firey, A. (coord.) A New History of Penance (pp. 19-71). Leiden y Boston: Brill. 
- Milliken, R., Shoup, L. H., y Ortiz, B. R. (2009). Oblone/Costanoan Indians of the San Francisco Peninsula and their Neighbors, Yesterday and Today. Oakland y San Francisco: Archaeological and Historical Consultants, National Park Service.

- Molina, A. (1984 [1565]). Confesionario mayor en la lengua mexicana y castellana. México: UNAM.

- Moreno, R. (1984). Introducción. En Molina, A. Confesionario mayor en la lengua mexicana y castellana (pp. 9-34). México: UNAM.

- Murillo Gallegos, V. (2009). Problemas de evangelización, problemas de traducción. Fray Juan Bautista de Viseo y sus textos para confesores, Nueva España (siglo XVI). Zacatecas: Universidad Autónoma de Zacatecas.

- Murillo Gallegos, V. (2010). Fray Juan Bautista de Viseo y sus Advertencias para los confesores de los naturales. En Viseo, J. B. Advertencias para los confesores de los naturales (pp. ix-xlvi). México: Los libros de Homero.

- Orrego González, F. (2014). La administración de la conciencia. Cultura escrita, confesión e ilustración en el mundo católico hispano a fines del Antiguo Régimen. Madrid: Universidad Complutense de Madrid, Facultad de Geografía e Historia, Departamento de Historia Moderna, Tesis de doctorado. Consultado en línea: http://eprints.ucm.es/29637/1/T35970.pdf, consultada en 12 de junio de 2017.

- Ricard, R. (2005). La conquista espiritual de México. Ensayo sobre el apostolado y los métodos misioneros de las órdenes mendicantes en la Nueva España de 15321524 a 1572, 2ª ed., 9ª reimp. México: Fondo de Cultura Económica.

- Sandos, J. (2004). Converting California: Indians and Franciscans in the Missions. New Haven y Londres: Yale University Press.

- Shipley, W. (1978). Native Languages of California. En Sturtevan, W. C. (editor). Handbook of North American Indians, vol. 8 (pp. 80-90). Washington D. C.: The Smithsonian Institution.

- Tejada y Ramiro, J. (1859). Colección de cánones y decretos de todos los concilios de la Iglesia de España y de América, vol. V. Madrid: Imprenta de D. Pedro Montero. 